Didáctica Geográfica nº 20, 2019, pp. 55-77

ISSN: 0210-492-X

D.L: M-3736-2014

\title{
PERSPECTIVAS DE FUTURO EN EL APRENDIZAJE DEL PAISAJE
}

Alfonso García de la Vega ${ }^{1}$

Recibido: 30/04/2019

Aceptado 31/07/2019

\section{RESUMEN:}

La revisión del currículum proporciona un enfoque educativo, cuanto menos sesgado, sobre el paisaje. Desde 2000, la aparición del Convenio Europeo de Paisaje y las sucesivas recomendaciones para orientar y concretar las intervenciones educativas se han obtenido algunos logros significativos. Entre otros, se han publicado proyectos de investigación y las recomendaciones dedicadas a la educación de los paisajes. El paisaje se convierte en aglutinador de los contenidos curriculares, bajo una perspectiva globalizadora e interdisciplinar. En este trabajo se muestran dos recursos didácticos, el itinerario didáctico y las geotecnologías, vinculadas con el conocimiento y valoración de los paisajes. El primero posee un recorrido disciplinar y educativo consolidado. Sin embargo, la tradición muestra diferencias temporales y espaciales significativas en cuanto al planteamiento metodológico. El segundo muestra una presencia más reciente y dominante. La aplicación de las tecnologías en el aula y en los itinerarios es un hecho. La incorporación de las geotecnologías en la vida ciudadana constituye un reto de futuro sobre la didáctica de la Geografía. Este trabajo muestra algunas miradas didácticas que guardan muchos de estos temas en relación con el paisaje. Asimismo, se ofrece una revisión sobre las investigaciones en la didáctica de la Geografía y sobre la literatura gris con el fin de sugerir nuevas líneas de investigación en la didáctica del paisaje y la aplicación en el aula.

1 Universidad Autónoma de Madrid.alfonso.delavega@uam.es 


\section{Palabras-clave:}

Paisaje, itinerario didáctico, ciudadanía espacial, geotecnologías,

\section{Abstract:}

The curriculum review provides an educational approach, the less skewed, about the landscape. Since 2000, the emergence of the European Landscape Convention and the successive recommendations to guide and specify educational complications have obtained some specific achievements. Among others, the research projects and recommendations dedicated to landscape education have been published. The landscape becomes the key of the curricular contents, under a globalizing and interdisciplinary perspective. In this paper there are two didactic resources, the fieldtrip and the Geotechnologies, linked to the knowledge and assessment of landscapes. The first has a consolidated disciplinary and educational path. However, the tradition shows temporal and spatial differences affected in terms of the methodological approach. The second shows a more recent and dominant presence. The application of technologies (IT) in the classroom and on the fieldtrip is a fact. The formation of Geotechnologies in citizen life constitutes a challenge of the future on the teaching Geography. This paper shows some didactic looks that keep many of these themes in relation to the landscape. Similarly, a review is offered on research in the teaching Geography and on gray literature in order to suggest new lines of research in landscape teaching and application in the classroom.

\section{KeYWORDS:}

Landscape Learning, Fieldtrip, Spatial Citizenship, Geotechnologies

\section{RESUMÉ:}

La révision du programme propose une approche pédagogique, la moins biaisée, du paysage. Depuis 2000, l'émergence de la Convention Européen du Paysage et les recommandations successives visant à orienter et préciser les interventions en matière d'éducation ont permis d'obtenir des résultats importants. Des projets de recherche et des recommandations consacrés à l'éducation au paysage ont notamment été publiés. Le paysage devient l'agglutinateur des contenus curriculaires, dans une perspective globalisante et interdisciplinaire. Ce travail présente deux ressources pédagogiques, l'itinéraire didactique et les géotechnologies, liées à la connaissance et à l'évaluation des paysages. Le premier a un parcours disciplinaire et éducatif consolidé. Cependant, la tradition montre d'importantes différences temporelles et spatiales dans l'approche méthodologique. La seconde montre une présence plus récente et dominante. 
L'application des technologies en classe et sur les itinéraires est un fait. L'intégration des géotechnologies dans la vie citoyenne est un défi pour l'avenir de l'enseignement de la géographie. Ce travail présente des aspects didactiques qui maintiennent nombre de ces thèmes en relation avec le paysage. De même, une revue de la recherche en enseignement de la géographie et de la littérature grise est proposée afin de suggérer de nouvelles pistes de recherche en matière d'enseignement et d'application du paysage en classe.

\section{MotS-CLÉ:}

Paysage, itinéraire didactique, citoyenneté spatiale, géotechnologies.

\section{PREÁmbUlo}

La comprensión y adquisición de los conceptos y destrezas geográficas conducen a alcanzar un perfil del ciudadano con pensamiento crítico y capacidad para comprometerse en las decisiones territoriales de la sociedad. Por ello, la alfabetización geográfica y la didáctica de la Geografía componen dos eslabones en la educación geográfica. La alfabetización consiste en el proceso de aprendizaje, que se refiere a la adquisición de las habilidades espaciales cotidianas, relacionadas con la elaboración de un trayecto (p.e., los itinerarios habituales desde casa al trabajo y los cambios realizados en el mismo debido al tiempo o a sucesos inesperados), con la lectura e interpretación de la información geográfica publicada en los medios de comunicación (p.e., la evolución de la población y su proyección de futuro en una pirámide de población) y la incorporación de los dispositivos móviles en las rutinas de la vida (p.e., la búsqueda de un lugar de ocio, o bien, la preparación de un viaje, utilizando la previsión del tiempo y la cartografía para elegir lugares de interés).

A lo largo de décadas, las líneas de investigación en la didáctica de la geografía han tratado de consolidar el conocimiento del entorno a través de numerosos recursos didácticos y estrategias metodológicas. Las investigaciones sobre el currículum de Geografía han demostrado que, los vaivenes en la legislación educativa y la estructura de los elementos curriculares prescriptivos, conducen a una Geografía enciclopédica y a un alejamiento de la realidad geográfica. El camino educativo de la geografía se aleja de la realidad y abandona la alfabetización de la ciudadanía.

En las últimas décadas, los medios de comunicación han invadido la realidad informativa. Tanto es así que han llevado a cabo una apropiación de los contenidos geográficos, que ha conducido a desdibujar la configuración del acervo geográfico. Entre estos contenidos se encuentran aquellos relacionados con la diversidad de los paisajes mundiales, el cambio climático, la realidad y las tendencias urbanas, la sostenibilidad del territorio, la despoblación de las áreas rurales y las principales vías de migración 
mundial, el uso de la cartografía en los dispositivos móviles, el aprovechamiento de los recursos naturales... Todo ello, pese a la aparición de algunos manifiestos de la Asociación Española de Geografía (AGE) dedicados a la despoblación y a la Amazonía, e incluso, sobre el derecho a la vivienda... aunque aún no ha aparecido ninguno sobre el cambio climático. También, la Unión Geográfica Internacional (UGI) redactó la Declaración Internacional sobre Educación Geográfica.

Todos esos temas candentes en la sociedad podrían abordarse desde una perspectiva interdisciplinar, y por supuesto, desde un enfoque geográfico. Así, la gestión y protección de los paisajes, e incluso su vulnerabilidad, la incidencia del cambio climático en las transformaciones territoriales, el planeamiento urbano en relación con la población, el diseño territorial de las vías de comunicación, los mercados de proximidad, la sostenibilidad del territorio vinculada a los recursos naturales y la distribución del poblamiento y la migración, la aplicación de la tecnología a las rutinas personales geoespaciales. Estos temas pertenecen al corpus de conocimiento de la Geografía y constituyen el "conocimiento frontera" de la disciplina.

En consecuencia, la Didáctica de la Geografía debiera ofrecer una respuesta educativa a estos temas. Las investigaciones de la Geografía y los manifiestos y declaraciones de las organizaciones geográficas, como las señaladas más arriba, debieran ser el referente disciplinar para la Didáctica. Así, la Didáctica podría orientar el currículum sobre las investigaciones geográficas y promover una adecuación rigurosa de los contenidos. En ambos casos, se trata de proponer una revisión de los contenidos del currículum, como en el segundo, adecuar las investigaciones geográficas al currículum, se precisa un vínculo entre los centros de investigación (universidades, institutos de investigación...) y los centros de enseñanza (institutos, escuelas...) y las futuras tendencias de la didáctica de la Geografía. En este sentido, en Australia, el National Committee for Geographical Sciences ha presentado un plan estratégico, donde se pretenden alinear la investigación y la enseñanza geográfica con la economía con el fin de afrontar estratégicamente los desafíos sociales, económicos y ambientales contemporáneos continentales (Australian Academic of Sciences, 2018).

Evidentemente, las habilidades memorísticas pueden ser utilizadas para aplicarse a situaciones reales. Sin embargo, "la educación superior", sentencia Unwin (1995, p. 285), "no debe ocuparse de inculcar hechos aceptados, sino de capacitar a los estudiantes para que desarrollen sus propios enfoques críticos ante el mundo en que viven". En definitiva, se trata de transmitir una formación crítica y el "deseo de adquirir conocimientos emancipadores", como afirma este mismo autor. Por tanto, la orientación de las estrategias didácticas proporciona nuevas posibilidades educativas desde la selección de los elementos curriculares, la reflexión sobre el planteamiento metodológico y la elección de los recursos didácticos. 


\section{REVISIÓN DE LOS CONTENIDOS GEOGRÁFICOS RELACIONADOS CON LA NORMATIVA DEL PAISAJE}

En España, a lo largo de décadas, la Didáctica de la Geografía ha consolidado el estudio del paisaje desde distintas perspectivas disciplinares y didácticas. En este artículo interesa abordar las aproximaciones didácticas al paisaje, sin perder de vista las aportaciones que se realizan desde la geografía y otras disciplinas al paisaje. El currículum sigue siendo el referente normativo en la educación, desde la perspectiva didáctica que requiere ajustarse a unos contenidos y estándares de aprendizaje. Ahora bien, el paisaje proporciona otro tipo de aproximaciones conceptuales y didácticas globales e interdisciplinares. Esto es, el currículum, como documento educativo que aborda el paisaje y representa la enseñanza formal, precisa completarse con estrategias metodológicas y recursos didácticos. Para ello, hay que recurrir a otra documentación, normativa e investigación educativa.

Por un lado, el Convenio Europeo del Paisaje (2000) y el Plan Nacional de Paisaje Cultural (2012) son los documentos más destacados en relación a la educación y sensibilización hacia el paisaje. La investigación educativa sobre el paisaje muestra diversas orientaciones didácticas, que proporcionan un marco de intervención en el aula a través de recursos metodológicos, como el itinerario didáctico, y recursos didácticos, como la cartografía. Todo ello permite avanzar en el conocimiento, las destrezas espaciales y la valoración de los paisajes. En este sentido, también hay que destacar la sensibilidad hacia los paisajes globales y locales, donde la identidad y el respeto sean valores hacia los paisajes ajenos y propios. Esto se refiere a la educación en paisajes identitarios y en paisajes hospitalarios, o bien, los paisajes donde se vive y aquellos donde se transitan y se viajan. Paisajes ajenos que pueden ser tan delicados y frágiles como los propios donde todos nos sentimos identificados.

Por otro lado, en esta última década ha aparecido un relevante conjunto de normativa estatal y europea. En Europa se ha constituido el Convenio Europeo de Paisaje (UE, 2000), de este Convenio han surgido otras disposiciones en relación con la educación para su desarrollo. En España, se han promulgado dos leyes: Ley 42/2007, de 13 de diciembre, de Patrimonio Natural y de la Biodiversidad y la Ley 45/2007, de 13 de diciembre, de desarrollo sostenible del medio rural. Estas dos leyes se promulgaron el mismo año 2007, que España ratificó el Convenio Europeo del Paisaje. Sin embargo, la normativa apenas recoge una referencia educativa explícita sobre el paisaje.

\subsection{La educación del paisaje en el Convenio Europeo de Paisaje}

En la última década, han aparecido dos normativas relevantes en relación con el paisaje. El Convenio Europeo del Paisaje (Unión Europea, 2000), en adelante CEP, y el Plan Nacional de Paisaje Cultural (2012) proporcionan un marco de referencia sobre 
la catalogación, conservación, divulgación... pero apenas existen referencias sobre el papel del paisaje en la educación. Algunos artículos del capítulo segundo del CEP se dedican a medidas específicas para los estados miembros de la Unión Europea sobre participación, sensibilización, formación y educación.

El CEP se propone establecer procedimientos de participación pública, donde confluya la cualificación experta y los valores atribuidos al paisaje por la población. Por tanto, la percepción social y las aspiraciones populares son claves de la participación ciudadana en relación al paisaje. En cuanto a sensibilización, el CEP pretende atender las relaciones entre las actividades de la vida cotidiana y las características del medio natural, el hábitat y las infraestructuras. La formación y educación de los ciudadanos sobre el paisaje constituye un binomio en el CEP, construido desde el fomento y desarrollo del conocimiento de los paisajes para todo el público. Dicho conocimiento debe alcanzar a todo el personal técnico administrativo, a la investigación aplicada, teórica e interdisciplinar.

El CEP pretende ofrecer una educación en los paisajes a partir del fomento, fortalecimiento y sensibilización de todas aquellas cuestiones que atañen a los paisajes. Se plantea un desarrollo educativo de los paisajes dirigido hacia todos los niveles educativos y, también, a través de la familia. El paisaje comenzaba a consolidar una proyección europea sobre unos contenidos dispersos en diferentes disciplinas, aunque todavía quedaba un camino por hacer. La publicación de nuevos documentos orientativos y consultivos aportan una valiosa dedicación al paisaje, desde una perspectiva educativa. Este es el caso de la recomendación europea CM/REC (2008)3 y CM/REC (2014)8 sobre las orientaciones para la aplicación del Convenio Europeo del Paisaje (Unión Europea, 2008 y 2014).

En 2008, se establecieron algunas orientaciones desde la Unión Europea para la aplicación del CEP. El apartado relacionado con la educación del CEP se refiere a la "participación, sensibilización, formación y educación" (Unión Europea, CM/REC, 2008, p. 46). Existe una referencia explícita referida a la complementariedad entre el conocimiento empírico de la población y el conocimiento científico. Esta dualidad conduce a la sensibilización por los paisajes y a la formación de la población, en especial a la enseñanza. En este sentido, las orientaciones europeas sobre el CEP buscan una formación entre paisaje y desarrollo sostenible, recursos naturales y equidad social (Unión Europea, CM/REC, 2008). En el caso de las Recomendaciones de 2014, el documento desarrollado proporciona algunas claves educativas de concreción para su aplicación en el aula. En 2009 y 2015, la Unión Europea publicó sendos documentos de recomendaciones sobre el paisaje (Unión Europea, 2009 y CM/REC, 2015). El primero constituye un documento de referencia sobre algunas orientaciones educativas del paisaje en la infancia y fue elaborado por Castiglioni. En el segundo se muestran 
estrategias didácticas sobre el paisaje en educación primaria y fue elaborado por Bovet, Pena y Ribas.

Desde la aparición del CEP en 2000, se han sucedido numerosos proyectos educativos y experiencias didácticas con repercusión en la literatura sobre la Didáctica de la Geografía. Batllori y Serra (2010) describen el proyecto titulado "Ciutat, territorio, paisatge" para reconocer una veintena de paisajes catalanes. Para ello, los autores hicieron unos trípticos con una imagen del paisaje de estudio, acompañado de cartografía, otras imágenes de detalle y actividades. El proyecto estaba dirigido al alumnado de educación secundaria y, además los profesores recibían formación para utilizar las carpetas con los paisajes en el aula.

Castiglioni (2010 y 2012) lleva a cabo diversas experiencias que le conducen a abordar el paisaje desde diversas perspectivas. Esta autora lleva a cabo un proyecto sobre los paisajes kársticos situados en los relieves de los bordes de la llanura padana, donde ofrece unas primeras pautas sobre la lectura del paisaje (Castiglioni, 2010). Estas pautas, a su vez, formarán una parte sustancial del estudio realizado sobre el Canale di Brenta para consolidar el Observatorio del Paisaje (Castiglioni y Varotto, 2013). Si el paisaje, como señala la misma autora, viniera a ser el instrumento educativo (Castiglioni, 2012), el Observatorio supondría el instrumento social. En este sentido, estas propuestas experimentales conducen a una aproximación educativa del paisaje a la sociedad, tal como propugnaba el CEP en 2000 y los documentos sobre orientaciones y recomendaciones, publicados posteriormente. En este momento, se llevan a cabo reflexiones sobre la educación del paisaje. Batllori y Serra (2017) apuestan por una educación del paisaje que conduzca a la enseñanza de la geografía y a una educación por la sostenibilidad.

Las recomendaciones dedicadas a la educación del paisaje, según la Unión Europea, deben comenzar en la familia y continuar a través de los planes de estudio en sus diferentes niveles educativos. Las orientaciones europeas apuntan hacia el aprendizaje de una lectura del paisaje, que supone entender las relaciones ecológicas, sociales y económicas con la ordenación del territorio. En el documento se afirma que "el paisaje constituye un recurso pedagógico porque, cuando lo interpretan, los alumnos se enfrentan cara a cara con los signos visibles de su marco de vida, que relacionan con las cuestiones de ordenación del territorio" (Unión Europea, CM/REC, 2008, p. 52).

\subsection{El paisaje diluido en el currículum}

Melcón Beltrán (1995) reflexiona sobre la organización curricular, que mantiene un enfoque piagetiano, que propone una jerarquía conceptual progresiva desde las estructuras más sencillas a las más complejas. O bien, las áreas disciplinares deben estar más ajustadas a las disciplinas académicas, o si, por el contrario, debe existir una 
organización basada en los temas relevantes de la actualidad o sobre los intereses del alumnado. Egan (1978) proporciona una visión sobre el currículum, centrada sobre los binomios conceptuales, dedicado a las grandes ideas de la civilización. Estos grandes temas, según el autor, podrían orientar una perspectiva diferente sobre el currículum, más aproximada a los intereses del alumnado.

Esta orientación curricular está bien afianzada en los países mediterráneos desde hace décadas. Sin embargo, hace décadas que en los países anglosajones se proponen otros enfoques curriculares. Así, Beane (1995) aboga por un enfoque integrado del currículo, en base a determinados contenidos disciplinares nucleares. Beane (2005) supone la implementación de una secuencia de contenidos pertinentes desde las diferentes materias. Así, se persigue la complementariedad de los contenidos específicos de cada disciplina a través de la elaboración de unos proyectos de trabajo que asimilen los numerosos elementos curriculares. Por consiguiente, habría que abordar desde una perspectiva integradora los temas relacionados con la gestión medioambiental, la sostenibilidad y la conservación de los paisajes. En la misma línea se pueden completar con las intervenciones humanas derivadas de sus usos y aprovechamientos realizados sobre el territorio, a fin de llevar a cabo un estudio sobre la evolución del paisaje.

De Miguel (2018b) reflexiona sobre la estructura curricular británica, pues proporciona una estructura sobre siete grandes ejes de contenidos conceptuales: lugar, espacio, escala, interdependencia, procesos físicos y humanos, medioambiente / desarrollo sostenible y diversidad cultural. Además de otros tantos dedicados a las habilidades geográficas estadísticas, gráficas y cartográficas incluyendo los SIG- el trabajo de campo, así como el proyecto escolar de investigación geográfica. Esta reflexión se dirige a replantear de manera decidida la normativa educativa, de manera que el marco político propugne un consenso social a fin de elaborar un currículo a largo plazo. De ahí se puede seleccionar los grandes contenidos y habilidades disciplinares y orientar hacia unas posibles líneas metodológicas y elección de recursos didácticos.

Casas y Erneta (2016) realizaron una revisión comparada sobre el paisaje en la Ley orgánica, 3 de mayo, de Educación (LOE, 2006) y Ley Orgánica de 8/2013, de 9 de diciembre para la Mejora de la Calidad Educativa (LOMCE, 2013). Estos autores resaltan el valor educativo del paisaje, la inclusión de los itinerarios en la formación de los paisajes, además de incluir otros recursos tecnológicos y cartográficos para su aprendizaje. Los autores consideran la protección y gestión del paisaje, además de proporcionarle los valores culturales, históricos y ecológicos, como parte del patrimonio. Martínez y García (2014) completan la revisión curricular sobre el paisaje en la normativa de la educación infantil más reciente en las diferentes comunidades españolas.

En ocasiones, tanto las disposiciones políticas como el devenir epistemológico de las disciplinas generan incertidumbre en el propio discurso geográfico plasmado en 
el currículum. Así ha sucedido, por ejemplo, con los contenidos sobre la ciudadanía y sobre el paisaje, que ha generado un discurso académico desde la didáctica y desde diversas disciplinas. El resultado origina una compleja adscripción de los elementos curriculares en la normativa. La propuesta de Fernández Caso (2007) recoge tres criterios orientadores del currículo: significatividad lógica y epistemológica, relevancia social, significatividad psicológica. Una propuesta que integraría las secuencias de contenidos geográficos sobre los mencionados criterios y proporcionaría un currículo integrado y articulado sobre dichos contenidos.

El análisis del currículum ha generado un considerable número de estudios sobre diferentes cuestiones educativas de la Geografía. Así, Gómez Ortiz (1993) consideraba necesario incorporar la polisemia del término paisaje en la didáctica de la Geografía. Este autor resaltaba el valor multidisciplinar por numerosas disciplinas, otorgándole diversos matices semánticos (equilibrio dinámico, energía, flujo...). El Convenio Europeo de Paisaje (2000) ha acotado el término de paisaje:

Por paisaje se entenderá cualquier parte del territorio tal como la percibe la población, cuyo carácter sea el resultado de la acción y la interacción de factores naturales y/ o humanos

Así, Gómez Ortiz (1993) hacía referencia a la aportación de la Institución Libre de Enseñanza y los movimientos de Renovación Pedagógica a lo largo del siglo XX. En la misma línea que la Institución de la que formó parte, Vidal Box (1976) expresaba que el profesorado debía transmitir la afición a la naturaleza, como parte de la observación directa de los fenómenos. Carandell (1925) realizó numerosos itinerarios, que quedaron publicados con sencillos bloques diagrama de los relieves representados. En el transcurso del siglo XX, naturalistas, como Dantín Cereceda, Hernández Pacheco y Solé Sabarís impulsarían un procedimiento metodológico a la Geografía Física, que influyó en la didáctica de la Geografía. Según Calaf (1991) el proceso consistiría en: observar registrar, deducir, comparar, clasificar...uso del mapa para contrastar la realidad y la realización de gráficos y esquemas. Un procedimiento que conduce al trabajo de campo, como clave en la investigación geográfica.

\section{EL PAISAJE EN EL TRABAJO DE CAMPO E ITINERARIOS DIDÁCTICOS}

Habitualmente, trabajo de campo e itinerarios didácticos son términos que vienen a reconocerse como similares en la literatura científica y educativa. En principio, las características de ambos son semejantes, pero su desarrollo y ámbito de aplicación 
educativa y académica resultan ser diferentes. Por ello, se va a intentar dilucidar los umbrales terminológicos de ambos conceptos. El trabajo de campo pertenece al corpus empírico de una disciplina, como es la Geografía. Martínez de Pisón (2009:21) afirma que "el geógrafo se formaba como observador, se adiestraba en leer la Tierra, leerla por contacto directo". La realización del trabajo de campo se lleva a cabo por un grupo de profesores y estudiantes para llevar a cabo una investigación, o bien, para contrastar los aprendizajes presentados en el aula con la realidad. Resulta obvio que las observaciones en el trabajo de campo abren nuevas miradas y las evidencias y datos obtenidos formulan nuevos interrogantes. Por ello, a modo de un laboratorio real y vivo, el paisaje muestra los elementos que son objeto de observación e identificación (roquedo, vegetación, poblamiento...). Estas observaciones pueden generar la toma de muestras, fotografías y otro tipo de evidencias para llevar a cabo un minucioso análisis. Así, el análisis del paisaje se completa con el análisis de las muestras en el laboratorio, que permitirá llevar a cabo una interpretación de los hechos geográficos que intervienen en la dinámica del paisaje.

Desde finales del siglo XIX, Giner de los Ríos promueve la intuición como medio de acercarse a la realidad, influido por la corriente krausista. Este proceso permite una aproximación tanto analítica como sintética de la realidad y, sobre todo, integral (Melcón Beltrán, 1991). Melcón Beltrán (1991) afirma que "excursiones escolares son el medio por excelencia de la educación intelectual. En esta misma línea, Souto González (2013) manifiesta que la identidad colectiva se consolida en el medio que se vive y a través de la adquisición de la concepción del espacio. Este autor considera que el aprendizaje en ámbito educativos no formales integra el conocimiento espacial y emocional del territorio. Mas áun, el mismo autor refleja que a lo largo de la historia curricular española el trabajo de campo sólo aparece reflejado en la LOGSE (1990). Por tanto, la salida de campo resulta clave en una educación integral de las personas. Sin embargo, en Geografía, la presencia curricular del trabajo de campo ha sido controvertida. Precisamente, en esa misma década de los noventa, el trabajo de campo se convierte en un requisito formativo en la educación obligatoria británica, según afirman Kent y Foskett (2000). Estos mismos autores señalan que el trabajo de campo, por diferentes causas, no es común en la educación geográfica en numerosos países, como Estados Unidos, China y Paises Bajos. Harvey (1991) apuntaba que la transferencia del conocimiento a través de los datos recogidos en el trabajo de campo... pueden ser una base para su discusión en el aula.

Bonnett (2008) expresa la propiedad de la geografía por llevar a los niños fuera del aula hacia las calles de la ciudad y los campos. El itinerario didáctico o geográfico constituye una elaboración preparada y diseñada para un eficaz proceso de aprendizaje. En este proceso resulta necesario contemplar los propios hitos, objeto de estudio, planteados en 
el itinerario como la estrategia didáctica planteada y los recursos didácticos empleados. La estrategia didáctica se refiere, por un lado, al planteamiento del itinerario en el cronograma educativo y por otro, la dinámica didáctica del itinerario.

Así, el itinerario precisa ser ubicado en la programación anual. Esta previsión de realizar el itinerario requiere la presentación del mismo, las tareas a realizar por el alumnado en el desarrollo in situ del itinerario y, por último, la realización de elaboración de un dossier. El dossier supondrá llevar a cabo la interpretación del paisaje, que servirá para la realización de la evaluación de toda la actividad. Además, esta planificación educativa del itinerario supone diseñar el papel de docentes y alumnado en el desarrollo del itinerario. Y, también, supone proporcionar los recursos didácticos del itinerario, como la cartografía en papel u offline.

Hammond (2018) afirma que el itinerario es un pilar en la educación geográfica, pues permite el desarrollo integral del geógrafo. El itinerario, afirma este autor, promueve el conocimiento y las destrezas geográficos y permite identificarse con el geógrafo. En esta misma línea, Lambert y Reiss (2016) proponen un conjunto de dimensiones educativas que se adquieren en el desarrollo del itinerario geográfico. En este sentido, estos autores resaltan el valor del lugar de estudio real, la aplicación y comprensión de destrezas contextualizadas, desarrollo de un aprendizaje en el mundo real real y, por último, las habilidades sociales.

El itinerario didáctico proporciona la adquisición de diversas destrezas geoespaciales, que forman parte del aprendizaje para toda la vida. Estas destrezas se organizan desde las más sencillas hacia las de mayor complejidad. La geografía promueve la aproximación a la realidad desde distintas perspectivas y escalas. Los itinerarios fomentan la adquisición de habilidades de orientación.... Las dimensiones y magnitudes proporcionan establecer analogías y diferencias entre contenidos geográficos, como altitudes y distancias, aplicados a paisajes mediante cálculos estimativos o absolutos (sierras y picos, o bien, caminos y campos de cereales). García Ruiz (1994 y 1997) y Liceras (2003 y 2013) llevaron a cabo un trabajo sistemático para observar e interpretar el paisaje desde un enfoque analítico. Castiglioni (2009) también promueve una lectura del paisaje mediante diversas etapas. Esta autora proporciona diversas lecturas para observar, identificar y describir los diferentes elementos bióticos, abióticos y emocionales del paisaje.

En esta misma línea de interpretación de los elementos del paisaje (Crespo, 2012). Serrano et al. (2016) aportan un renovado pautado de identificar, discriminar y definir los hitos que estructuran el itinerario didáctico. También hay que considerar los itinerarios y trabajos de campo destinados a reorientar el enfoque geográfico sobre unos determinados contenidos. En este sentido, García Hernández et al. (2019) abordan un itinerario sobre los riesgos de los aludes, o bien, Jerez García y Serrano de la Cruz (2017) 
orientan un itinerario sobre los paisajes degradados por la actividad humana. Asimismo, los itinerarios didácticos han proporcionado planteamientos docentes definidos por un determinado dominante educativo. Dole et al, (2016) ofrecen ese cambio pedagógico que centra el interés del aprendizaje en el estudiante. El mismo modelo, que según García Pérez (2000), puede aplicarse al aula de Geografía. En consecuencia, el enfoque constructivista reside en propuestas de aprendizaje basados en problemas (Bradbeer, 1996; Savery y Duffy, 2001 y García de la Vega, 2012), el uso del flipped classroom (Jericó y Erneta, 2017), o bien, a través de los recursos didácticos para la educación primaria y planteados por Bovet et al. (2015).

Caton (2006) examina las formas de aprendizaje en el trabajo de campo, como formas de aprendizaje del mundo real. Este autor ofrece cinco tipos de trabajo de campo, dependiendo del grado de autonomía del estudiante en las actividades (desde el profesor que interpreta el paisaje hasta la realización de pequeñas investigaciones hasta realizar pequeños descubrimientos) y del tipo de actividad a realizar (a partir de hipótesis, investigaciones, problemas y estudios de caso). Por el contrario, Fuller et al. (2006) cuestionan el aprendizaje efectivo del trabajo de campo en Geografía. En esta investigación realizada en universidades de tres continentes, estos autores resaltan comprobar tres supuestos del valor del trabajo de campo: experiencia en el mundo real, desarrollo de habilidades, transferibles y técnicas, y beneficios sociales. Los impactos sobre la efectividad del aprendizaje en el campo pueden variar desde los imprevistos del trabajo de campo (las condiciones meteorológicas) hasta cuestiones culturales y didácticas (enfoque metodológico referido a todo el proceso de preparación de la salida).

En suma, tanto el itinerario didáctico como el trabajo de campo están consolidados como técnicas de aprendizaje, pertenecientes a estrategias y metodologías pedagógicas en los diferentes niveles educativos. Ahora bien, la proliferación del itinerario, tanto en los ámbitos de aprendizaje formal como no formal, no confirma su éxito didáctico. Por ello, uno de los retos educativos de la enseñanza de la Geografía reside en revisar los factores didácticos que determinan el itinerario, así como el planteamiento metodológico y la evaluación de los aprendizajes alcanzados. Entre los factores señalados se encuentran los condicionantes imprevisibles que alteran el plan del itinerario, la identidad cultural del grupo de estudiantes con el paisaje estudiado, el compromiso del alumnado en implicarse en el proceso de aprendizaje a través del itinerario... La revisión del planteamiento metodológico requiere comprobar la idoneidad de la autonomía del estudiante en el itinerario, el planteamiento de una forma de aprendizaje a través de investigación o problemas, por ejemplo, o bien, la dinámica grupal empleada. Por último, se considera que haya que incorporar algún tipo de evaluación tanto en las distintas fases de la realización del itinerario didáctico como al final del mismo. 


\section{RETOS DE APRENDIZAJE SOBRE EL PAISAJE EN EDUCACIÓN GEOGRÁFICA}

En la educación geográfica, el paisaje constituye uno de los contenidos más integradores y aglutinadores de otros temas. E incluso, se pueden abrir interrelaciones con contenidos asociados a otros ámbitos de conocimiento. En este apartado se pretenden abordar dos de esos temas que podrían vincularse con los contenidos de paisaje y con una firme proyección de futuro en la educación geográfica. Los temas elegidos son: por un lado, la ciudadanía y, por otro, el desarrollo del pensamiento espacial y la cartografía. Ambos contenidos se ofrecen en relación con el paisaje. Aún quedan otros retos para abordar en el futuro, como la sostenibilidad, la despoblación, el cambio climático y la inestabilidad de los océanos, por citar algunos de ellos.

El estudio del paisaje se ha servido de los sistemas de información geográfica y de las geotecnologías para la identificación, clasificación, análisis, síntesis y representación del territorio. Sin embargo, la perspectiva didáctica proviene desde el conocimiento, la percepción y la vivencia de los paisajes. Para ello, la didáctica del paisaje promueve la adquisición de las habilidades geoespaciales y el fomento de las destrezas en las aplicaciones de los dispositivos móviles. Estos vínculos disciplinares entre los estudios disciplinares del paisaje y las tecnologías geoespaciales aplicadas a la didáctica constituye uno de los retos educativos de la Geografía. Antes que esto, existen algunas experiencias e investigaciones, que han abordado estos contenidos.

\subsection{La ciudadanía y la ciudadanía espacial en el paisaje}

¿No podría abordarse aquí los compromisos de la ciudadanía con el paisaje y como éste interviene en el equilibrio global? Así, por ejemplo, la evaluación de un paisaje natural conduce tanto a revisar las claves del cambio climático como a los referidos sobre la sostenibilidad. Y, aún más, también pueden encontrarse vestigios de dicho equilibrio en los paisajes urbanos y rurales. El proceso de transformación de los residuos sólidos aparece como un problema clave en la gestión de las ciudades. La inmigración y la despoblación conduce a los procesos de desequilibrios territoriales en la propiedad ý explotación de la tierra en los paisajes rurales. Por tanto, resulta necesario definir el compromiso de la ciudadanía, entendiendo como tal las personas que habitan el mundo (no sólo las ciudades).

No obstante, la ciudadanía podría participar en la elaboración de una historia del paisaje, donde las experiencias particulares, como las vivencias ajenas sobre un mismo paisaje ofrezcan una visión contrastada de ese paisaje. En un mundo globalizado, donde la movilidad de las personas resulta ser una clave del análisis geográfico, las impresiones personales de los paisajes de vida, sean de alojamiento hospitalario o rechazo, como de 
tránsito y turismo proporcionan una mirada personal de los paisajes mundiales. Para ello, por un lado, habría que recurrir a las percepciones de la literatura, la cartografía, la historia y el arte, mediante las fuentes de documentación, para reconocer e interpretar las miradas de los paisajes (García de la Vega 2016 y 2018 b y Martínez de Pisón, 2017).

Además, la ciudadanía podría elaborar sus relatos narrativos, siguiendo las pautas de Bruner (1991), o bien, las historias de mapas, storymaps, registradas por ESRI. Bruner (1991) sostiene que la experiencia y la memoria de los acontecimientos humanos se registra, principalmente, en forma de historias narrativas, Estas narraciones derivan en una variedad de formas expresivas y formales. Bruner (1991) considera que las narrativas son una versión de la realidad, regidas por la aceptación convencional y la propia necesidad expresiva, más que estructura lógica y empírica de la realidad. Por consiguiente, la narrativa se convierte en un instrumento de aproximación sobre el paisaje percibido.

García Pérez y Alba (2007) afirman que la participación ciudadana resulta esencial para llevar a cabo el desarrollo de la educación para la ciudadanía. Si bien, los autores, en base a la realización de unas experiencias comprueban que resulta muy complicado que los ciudadanos participen en las decisiones. Sin embargo, parece que Donert et al. (2019) consideran que es posible fomentar el empoderamiento juvenil para la participación democrática. El uso de las Geo-TIC promueve la toma de decisiones en una sociedad juvenil democrática y transformadora.

Piotrowska et al.(2019) muestran el interés de las competencias ajenas a las disciplinas, que denominan "blandas". Entre estas competencias se encuentran: la comunicación, el valor de la expresión, la autoestima y la responsabilidad. Sin embargo, estas competencias deben acompañarse de conceptos educativos específicos, como son: educación científica basada en la investigación, método de proyectos, trabajo de campo, senderos educativos geográficos, uso de tecnologías de geoinformación y geoespaciales. Sin embargo, como se aborda más adelante, parece conveniente estructurar las habilidades de la información y representación geoespacial de los estudiantes. Dado el auge alcanzado por los medios digitales en la sociedad, tal vez, se pueda ofrecer una perspectiva educativa sobre las competencias ciudadanas.

En suma, la participación social parece constituir un objetivo educativo a largo plazo. Pues se trata de fomentar una educación para la ciudadanía, que conduzca a la participación en los procesos sociales decisivos. En las próximas décadas se espera que aumente el compromiso de la participación ciudadana con formación crítica, que intervenga en los procesos sociales y económicos que definen las transformaciones de los paisajes. Así, la despoblación y el cambio climático formará parte de la toma de decisiones a diferentes escalas participativas. 


\subsection{El pensamiento espacial y las geotecnologías aplicadas al paisaje}

El National Research Council (2006) define el pensamiento espacial como una combinación constructiva de habilidades cognitivas compuesta por los conceptos de espacio, el uso de herramientas de representación y la aplicación de procesos de razonamiento. Hay numerosos conceptos espaciales, como sentido, dirección, localización, proximidad, dimensión, escala, continuidad, patrón y red que configuran el conjunto de las habilidades geoespaciales. Ahora bien, siempre bajo la perspectiva geográfica, tal como señala Massey (1984) el espacio no se aleja de la realidad, pues es una construcción social. Y, por tanto, el espacio, en cuanto que concepto social y abstracto, enlaza con la elaboración de los paisajes, como concepto social y concreto, interrelacionándose los procesos naturales y las intervenciones humanas.

En el currículo español, la cartografía aparece como un bloque de contenidos desarrollado a lo, largo del tercer curso de primaria. Sin embargo, el desarrollo y adquisición del pensamiento espacial se orienta, principalmente, hacia la identificación y localización de lugares (Breda et al., 2015). De Miguel (2018a) ha revisado el currículum de la educación secundaria, donde aparece sesgada y asociada a la historia. Por tanto, en ambas etapas, el currículo registra un escaso interés formativo por la cartografía, como medio para representar, interrelacionar e interpretar los hechos geográficos. Hace décadas, Unwin (1992) afirmaba que la responsabilidad educativa de la Geografía de ofrecer una interpretación crítica de la ocupación humana y de las diferencias entre los lugares. Para ello, el mismo autor apunta hacia la interpretación del currículum oficial, para así orientar la Geografía hacia la reflexión sobre cuestiones relevantes de la sociedad contemporánea, como el deterioro ambiental, el cambio climático y el acceso desigual a los recursos naturales.

Hay diferentes niveles de adquisición del pensamiento espacial. La relación establecida entre el tipo de espacio vivido, percibido y concebido en relación con la etapa de pensamiento concreto y formal no resulta abstracta. Desde edades tempranas, los niños exploran el espacio que les rodea gateando hasta andar. Posteriormente, los niños comienzan a explorar su entorno próximo en base al desarrollo de determinadas capacidades geoespaciales, vinculadas a la adquisición y aplicación de conceptos espaciales, como delante-detrás, izquierda-derecha... recto-de frente, hacia arribaabajo...) según Kastens y Liben (2010). En la tercera etapa, los niños transcienden el espacio al mundo abstracto e incorporan la representación cartográfica y matemática, según los artículos mencionados más arriba.

La lectura y comprensión del lenguaje cartográfico para representar el espacio permite desenvolverse en el mismo y adquirir unas destrezas relacionadas con la vida cotidiana. Unas habilidades espaciales, junto al uso de las aplicaciones geotecnológicas, que permiten un aprendizaje para toda la vida (García de la Vega, 2019). En este sentido, 
la adquisición de las capacidades geoespaciales para el aprendizaje para toda la vida es necesaria para moverse en el espacio y enfrentarse a las situaciones reales del mundo.

Una forma de desarrollar el pensamiento espacial pasa por el desarrollo lúdico de las habilidades geoespaciales. Marrón Gaite (1996, 2002 y 2005), Breda y García de la Vega (2018) y Zecha (2019) promueven diferentes tipos de juegos de simulación, analógicos manipulables o mediante el geocatching a través de una enseñanza activa lúdica, tanto dentro como fuera del aula. Warburton y Higgitt (1997) aportan dos estudios de caso para comprobar el uso de las tecnologías aplicado a la preparación de los trabajos de campo. Las tecnologías proporcionan un conocimiento previo sobre el área geográfica del trabajo de campo.

Gersmehl y Gersmehl (2007) señalan que la localización, siendo necesaria e importante el desarrollo de otros rasgos del razonamiento espacial. Estas características están arraigadas al objeto de la Geografía, pues a través de condiciones y conexiones entre lugares se realizan los estudios de los mismos. Estos rasgos se refieren a la comparación, proximidad, región, secuencia, jerarquía, analogía, modelo y asociación. Numerosos estudios muestran la capacidad espacial para asimilar la información procedente de los mapas topográficos y fotografías aéreas (Uttal, 2000, Plester et al., 2002 y Rapp et al.2007) desde edades tempranas. Este hecho indica que, si los elementos curriculares están bien diseñados y organizados a lo largo de las etapas escolares se impulsan las capacidades geoespaciales.

Partoune y Merenne-Shoumaker formularon diversas competencias geográficas, disciplinares y transversales, respectivamente. Entre estas competencias, Partoune define numerosas competencias relacionadas con las capacidades espaciales como, por ejemplo, construir mapas y croquis o elaborar modelos espaciales a diferentes escalas. MerenneSchoumaker propone, entre otras competencias transversales, construir una síntesis de diferentes formas (sistémica, gráfica, esquemática) (citadas en García de la Vega, 2018a)

En esta línea, si las capacidades geoespaciales son vitales en el desarrollo humano, Gryl et al. (2010) proponen el término, la ciudadanía espacial, como esa capacidad para utilizar la información espacial y las tecnologías geoespaciales. Kanwischer et al. (2012) se plantean, bajo este mismo término, la propuesta de un conjunto de competencias de ciudadanía espacial, incluidas en el currículum. Para ello, estos autores se proponen revisar las claves educativas de la tecnología convencional, desde la reflexión, comunicación y participación para elaborar unas competencias curriculares de la ciudadanía espacial en la educación secundaria. En esta misma línea, Shin y Bednarz (2019) promueven el estudio de la ciudadanía espacial desde la necesidad de la geografía para preparar a la sociedad en el acceso a las tecnologías geoespaciales. Para ello, las autoras proponen el dominio del espacio, el lugar, la escala... y las relaciones humanas que intervienen en todos los ámbitos de la sociedad. 
Posiblemente, tal como se ha señalado más arriba, el trabajo de campo se configura como una de las formas de aproximación a la realidad y los sistemas de información geográfica proporcionan los recursos de análisis, interpretación y síntesis de esa misma realidad... Por consiguiente, trabajo de campo y tecnología constituyen dos piezas clave del currículum en la enseñanza de la Geografía. Ambas permiten abordar el conocimiento del paisaje desde distintas perspectivas didácticas, aunque con el mismo objetivo. Esto es, obtener una lectura prolija de sus componentes para su tratamiento informativo y gestión del territorio mediante la aplicación de las geotecnologías, orientada hacia la formación de la ciudadanía espacial y del pensamiento crítico.

\section{CONCLUSIONES}

El paisaje representa un lugar central en el aprendizaje de la geografía. El paisaje constituye un contenido aglutinador, donde se interrelacionan numerosos contenidos disciplinares e interdisciplinares. La limitación ofrecida por el currículum conduce a encontrar posibles soluciones educativas, ya sea desde perspectiva metodológica o de los recursos didácticos. Una de ellas concierne a la integración de los contenidos relacionados con el paisaje en la organización curricular. La normativa estatal y europea sobre el paisaje proporciona algunas pautas de reconocimiento del paisaje, natural y cultural. También las figuras de los parques nacionales, parques naturales y geoparques puede proporcionar nuevas miradas para llevar a cabo el aprendizaje sistemático sobre el paisaje. Sin embargo, aún queda un recorrido para concretar muchas de sus propuestas en el nivel educativo.

En este artículo se revisa el itinerario didáctico, al ser clave educativa en el aprendizaje del paisaje. El itinerario posee un dilatado recorrido pedagógico, cuyas fases didácticas parecen estar consolidadas en la didáctica de la Geografía. Sin embargo, todavía queda la revisión del entorno de aprendizaje y la aplicación de las tecnologías geoespaciales. Así se puede llevar a cabo la primera aproximación al desarrollo de las habilidades geoespaciales a través de la cartografía y de las tecnologías aplicadas al paisaje. En este sentido, la ciudadanía, que ha sido un tema clave en las dos últimas décadas, adquiere un nuevo valor. Uno de los retos consiste en construir una ciudadanía espacial, donde se muestra el valor de las tecnologías en la vida cotidiana, su uso y su compromiso en la vida social.

\section{BIBLIOGRAFÍA}

Australian Academic of Sciences, 2018. Geography Shaping Australia's Future, Canberra: National Comittee for Geographical Sciences.

Batllori Obiols, R. y Serra i Sala, J. M. 2010. El proyecto "Ciutat, territorio, paisatge": un recurso innovador para la educación del paisaje en la enseñanza secundaria, Iber: Didácticas de las ciencias sociales, geografía e historia, $\mathrm{n}^{\circ}$ 65, pp. 17-26. 
Batllori Obiols, R. y Serra i Sala, J. M. 2017. D’ensenyar geografía a través del paisatge a educar en paisatge, Documents d'Anàlisi Geogràfica, vol. 63/3, pp.617-630.

Beane, J. A. 1995. Curriculum Integration and the Disciplines of Knowledge, The Phi Delta Kappan International, vol. 76, nº 8, pp. 616-622.

Beane, J. A. 2005. La integración del currículum. Madrid: Morata.

Bonnet, A. 2008. What is Geography? London: SAGE Publications Ltd.

Bovet, M.T., Pena, R. y Ribas, J., 2004. "El paisaje como recurso educativo en el marco de la educación para la participación”. Didáctica Geográfica, 2a época, 6, pp. 33-48.

Bradbeer, J. 1996. "Problem-based learning and fieldwork: a better method of preparation? "Journal of Geography in Higher Education, vol. 20, n. 1, pp. 11-18.

Breda, T. V. y García de la Vega, A. 2018. "El desarrollo del razonamiento geográfico a través de una propuesta ludo-didáctica en la ciudad", Didáctica Geográfica, n. 19, pp. 197-220.

Breda, T. V., García de la Vega. A. y Straforini, R. 2015. "Raciocínio espacial no ensino de Geografia. Uma análise da Cartografia nos documentos curriculares da Espanha e Brasil". In: Sebastiá Alcaraz, R. y Tonda Monllor, E. M. (Coords.): Investigar para innovar en la enseñanza de la Geografía. Alicante: CEE, pp. 933-958.

Bruner, J. (1991). "The Narrative Construction of Reality", Critical Inquiry, v. 18, n. 1, pp. 1-21

Calaf i Masachs, R. 1991. L'ensenyament de la geografia a l'escola, Barcelona: Barcanova Educació.

Carandell Pericay, L. 1925. "La Sierra de Cabra, centro geográfico de Andalucía", Conferencia leída en el Instituto Nacional de Segunda Enseñanza de Córdoba el 31 de marzo de 1925

Castiglioni, B. 2009. Educare al Paesaggio, Montebelluna: Museo di Storia Naturale e Archeologia.

Castiglioni, B. 2010. "La experiencia educativa en el paisaje: el proyecto 3KCL", Iber: Didácticas de las ciencias sociales, geografía e historia, n ${ }^{\circ} 65$, pp. 44-55.

Castiglioni, B. 2012. "Il paesaggio coem strumento educativo", Educación y futuro: revista de investigación aplicada y experiencias educativas, vol. 27, pp. 51-65.

Castiglioni, B. y Varotto, M. 2013. Paesaggio e osservatori local. L'esperienza del Canale di Brenta, Milano: Franco Angeli..

Caton, D. 2006. "Real world learning through geographical fieldwork". En: D. Balderston (Ed.) Secondary Geography Handbook, Sheffield: Geographical Association, pp. 60-73. 
Crespo Castellanos, J. M., 2012. "Un itinerario didáctico para la interpretación de los elementos físicos de los paisajes de la Sierra de Guadarrama". Didáctica Geográfica, n. 13, pp. 15-34.

De Miguel González, R. 2018a. "El currículo de geografía en España: evolución y tendencias actuales". En: García de la Vega, A. (Ed.): Reflexiones sobre educación geográfica. Revisión disciplinar e innovación didáctica. Madrid: Ediciones de la UAM y Ediçoes LAGIM, 2018. pp. 191-215.

De Miguel González, R. 2018b. "Geografía y tiempo contemporáneo: educación geográfica y enseñanza de las ciencias sociales para el mundo global", Revista de Investigación en Didáctica de las Ciencias Sociales (REIDICS), n. 2, pp. 36-54.

Dole, S.; Bloom, L. y Kowalske, K. 2016. "Transforming Pedagogy: Changing perspectives from teacher-centered to learner-centered". Interdisciplinary Journal of Problem-Based Learning, vol. 10 (1), pp.

Donert, K.; De Miguel González, R. y Luppi, A. 2019. "YouthMetre: Open Data to Empower Young People to Engage in Democracy and Policymaking". In: De Miguel, R., Donert, K. \& Koutsopoulos, K. (Eds.): Geospatial Technologies in Geography Education, Cham: Springer, pp.87-101.

Egan, K. 1978. "What's Curriculum”, Curriculum Inquiry, vol. 8, n 1, pp. 66-72.

Fernández Caso, M. V. 2007. "Discursos y prácticas en la construcción de un temario escolar en geografía”. En: Fernández Caso, M. V. y Gurevich, R. (2007). Geografía: nuevos temas, nuevas preguntas. Buenos Aires: Biblos, pp. 17-36

Fuller, I; Edmondson, S.; France, D. Higgitt, D. y Ratinen, I. 2006. "International Perspectives on the Effectiveness of Geography Fieldwork for Learning", Journal of Geography in Higher Education, vol. 30, n. 1, pp. 89-101.

García Hernández, C.; Ruiz Fernández, J. y Rodríguez Gutiérrez, F. 2019. "El fenómeno de los aludes a través de un itinerario didáctico en la Montaña Cantábrica". Cuadernos geográficos de la Universidad de Granada, vol. 58, n. 2, pp. 126-151.

García Perez, F. F. 2000. Los modelos didácticos como instrumento de análisis y de intervención en la realidad educativa, Biblio $3 W$. Revista Bibliográfica de Geografía y Ciencias Sociales, no 207, 18 de febrero de 2000.

García Perez, F. F. y Alba Fernández, N. de 2007. Educar en la participación como eje de una educación ciudadana. Reflexiones y experiencias, Didáctica Geográfica, vol. 9, pp. 243-258.

García Ruiz, A. L. 1994. Los itinerarios didácticos: una de las claves para la enseñanza y comprensión de la Geografía, Iber: Didácticas de las ciencias sociales, geografía e historia, $\mathrm{n}^{\mathrm{o}} 1$, pp. 117-126. 
García Ruiz, A. L. 1997. El proceso de desarrollo de los itinerarios geográficos, Didáctica Geográfica, $\mathrm{n}^{\circ}$ 2, pp. 3-10.

Garcia de la Vega, A. 2012. "El aprendizaje basado en problemas en los itinerarios didácticos vinculados al patrimonio". Educación y futuro: revista de investigación aplicada y experiencias educativas, n. 27, pp. 155-175.

García de la Vega, A. 2016. "La narración del paisaje y su relación con la adquisición de los conceptos geográficos". En: Castrogiovanni, C. (Ed.): Movimentos para ensinar geografia - oscilações, Porto Alegre (Brasil): Letra 1, pp. 17-30,

García de la Vega, A. 2018a. "Análisis del currículo español. Perspectiva desde la geografía y su didáctica”, Revista Brasileira em Educação de Geografía, vol. 8, n. 5, pp. 5-38.

García de la Vega, A. 2018b. "Planteamiento didáctico sobre los conceptos geográficos en torno a la ciudad en los diarios de viaje", En: H. C. Callai; T. D, de Oliveira y C. Copetti (Orgs.): A cidade para além da forma, Curitiba (BR): CRV, pp. 129-143.

García de la Vega, A. 2019. "Spatial Thinking Ability Acquisition through Geospatial Technologies for Lifelong Learning”. En: De Miguel, R., Donert, K. \& Koutsopoulos, K. (Eds.): Geospatial Technologies in Geography Education, Cham: Springer, pp.21-40.

Gersmehl, P. J. y Gersmehl, C. A. 2007. "Spatial thinking by young children: Neurologic evidence for early development and 'educability"'. Journal of Geography, 106(5), pp.181-191.

Gómez Ortiz, A. 1993. "Reflexiones acerca del contenido "paisaje" en los "currícula" de la enseñanza obligatoria", Revista Interuniversitaria de Formación de Profesorado, no 16, pp. 231-240.

Gryl, I. y Jekel, T. y Donert, K. 2010. "GI and Spatial Citizenship”. En: Jekel, T., Koller, A., Donert, K. \& Vogler, R.: Learning with Geoinformation V-Lernen mit Geoinformation V, Berlin: Wichmann Verlag

Hammond, L. 2018. "The place of fieldwork in geography education”. In: Mark Jones and David Lambert: Debates in Geography Education, Abingdon, Oxon (GB): Routledge.

Jerez García, Ó. y Serrano de la Cruz Santos-Olmo, M. A. (2016). "El interés didáctico de los paisajes alterados: la Reserva de la Biosfera de La Mancha húmeda (España) como ejemplo de estudio", Contexto \& Educação, vol. 31, n.99, pp. 52-80.

Jericó Casas, M. y Erneta Altarriba, L. 2017. "Propuesta didáctica para la enseñanza del paisaje a través del empleo de la metodología Flipped Classroom", Publicaciones Didácticas, 78, pp. 448-498. 
Kastens, K. A. y Liben, L. S. 2010. "Children's strategies and difficulties while using a map to record locations in an outdoor environment". Journal International Research in Geographical and Environmental Education, vol. 19, n 4, pp. 315340 .

Kanwischer, D.; Schulze, U. y Gryl, I. 2012. "Spatial Citizenship - Dimensions of a Curriculum”, En: Jekel, T., Car, A., Strobl, J. y Griesebner, G. (Eds.): GI_Forum 2012: Geovizualisation, Society and Learning. Berlin: Herbert Wichmann Verlag, pp. 172-181.

Kent, A. y Foskett, N. 2000. "Fieldwork in the school Geography curriculum-pedagogical issues and development". En: R. Gerber and G.K. Chuan (eds.): Fieldwork in Geography: Reflections, Perspectives and Actions, Dordrecht: Kluwer Academic Pubs pp. 171-193.

Lambert. J. 2010. Digital storytelling cookbook. Berkeley, CA: Digital Diner Press.

Lambert y Reiss 2016. "The place of fieldwork in geography qualifications”, Geography, vol. 101 (1), pp. 28-34.

Liceras, A. 2003. Observar e interpretar el paisaje: Estrategias didácticas. Granada, Grupo Editorial Universitario.

Liceras, A. 2013. "Didáctica del paisaje". Íber. Didáctica de las Ciencias Sociales, Geografía e Historia, 74, 85-93.

Marrón Gaite, M. J. 1996. “Los juegos de simulación”, Didáctica Geográfica, nº 1, pp. 45-56.

Marrón Gaite, M. J. 2002. "Desarrollo de la orientación espacial en la educación infantil a través del juego", En: Barreales Llamas, M.; Moreno Martín, M. C. y Gervilla Castillo,Á. M.(Coord.): Necesidades educativas de la infancia ante el nuevo milenio, Málaga: Centro de Ediciones de la Diputación de Málaga, vol. 2, pp. 919-926.

Marrón Gaite, M. J. 2005. "Metodología lúdica y aprendizaje significativo en Geografía propuesta de un juego de simulación para la educación intercultural”, Didáctica geográfica, $\mathrm{n}^{\circ} .7$, pp. 383-406.

Martínez de Pisón, E. 2009. La belleza del oficio de geógrafo. Madrid: Ediciones de la UAM.

Martínez de Pisón, E. 2017. La montaña y el arte, Madrid: Ércola Ediciones.

Martínez, R., y García, R, 2014. "El concepto paisaje en los currícula de Educación Infantil de las Comunidades Autónomas Españolas" en R. Martínez Medina y E. M. Tonda Monllor, eds. Nuevas perspectivas conceptuales y metodológicas para la educación geográfica. Córdoba: Grupo de didáctica de la Geografía (A.G.E.) y Universidad de Córdoba, pp. 479-495. 
Massey, D. 1984. “Geography matters!”. En: D. Massey y J. Allen (eds.): Geography matters. A Reader, Cambridge: Cambridge University Press.

Melcón Beltrán, J. 1991. "Las excursiones escolares y la educación integral”, Estudios Geográficos, t. LII, no 203, 239-261.

Melcón Beltrán, J. (1995). Renovación de la enseñanza de la Geografía en los orígenes de la España contemporánea, Madrid: Ediciones de la Universidad Autónoma de Madrid.

National Research Council. 2006. Learning to think spatially: GIS as a support system in the K-12 curriculum. Washington, DC: The National Academies Press.

Piotrowska, I.; Cichoń, M.; Abramowicz, D. y Sypniewski, J. 2019. Challenges in Geography Education. A Review of Research Problems, Quaestiones Geographicae, 38(1), 71-84

Plester, B.; Richards, J.; Blades, M. y Spencer, Ch. 2002. "Young Children's Ability to Use Aerial Photographs as Maps”, Journal of Environmental Psychology, vol. 22, pp. 29-47.

Rapp, D. N.; Culpepper, S. A.; Kirkby, K. y Morin, P. 2007. "Fostering Students' Comprehension of Topographic Maps", Journal of Geoscience Education, v. 55, n. 1 , pp. 5-16.

Savery, J. R. y Duffy, Th. M. (2001). Problem-based Learning. An instructional model and its constructivist framework, Centre for Research on Learning and Technology, Technical Report, vol. 16, $\mathrm{n}^{\mathrm{o}}$ 1, Bloomington: Indiana University.

Shin, E. E. y Bednarz, S. W. (2019). Spatial Citizenship Education, New York: Routledge. Serrano de la Cruz Santos-Olmo, M. A.; García Rayego, J. L. y Jerez García, Ó. (2016). Propuesta preliminar para la identificación de Lugares de Interés Didáctico en espacios naturales protegidos, Didáctica Geográfica, n 17, pp. 159-176.

Souto González, X. M. (2013). Didáctica de la Geografía y currículo escolar. En: De Miguel González, R.; De Lázaro y Torres, M. L. y Marrón Gaite, M. J. (Coord.): Innovación en la enseñanza de la Geografía ante los desafíos sociales y territoriales. Zaragoza: Institución “Fernando el Católico” (CSIC), pp. 121-146.

Unión Europea. 2000. Convenio Europeo del Paisaje. Florencia.

Unión Europea. 2008. Recomendación CM/REC (2008)3 del Comité de Ministros a los Estados miembros sobre las orientaciones para la aplicación del Convenio Europeo del Paisaje. Bruselas.

Unión Europea. 2009. 5th Council of Europe Conference on the European Landscape Convention. Education on Landscape for Children. Estrasburgo. 
Unión Europea. 2014. Recommendation CM/Rec (2014)8 of the Committee of Ministers to member States on promoting landscape awareness through education. Bruselas.

Unión Europea. 2015. Recommendation CM/Rec (2015) of the Committee of Ministers to member States on pedagogical material for landscape education in primary school. Estrasburgo.

Uttal, D. H. 2000. "Seeing the big picture: map use and the development of spatial cognition", Developmental Science, vol. 3, $\mathrm{n}^{\circ}$ 3, pp 247-286.

Unwin, T. 1992. El lugar de la Geografía, Madrid: Cátedra.

Vidal Box, C. 1976. Guía de recursos pedagógicos en Madrid y sus alrededores. Madrid: Consejo Superior de Investigaciones Científicas.

Warburton, J. y Higgitt, M. (1997). Improving the Preparation for Fieldwork with IT: two examples from Physical geography, Journal of Geography in Higher Education, vol. 21, n. 3, pp. 333-347.

Zecha, S (2019). EarthCaching as a Possible Way to Raise Enviromental Awareness, En: De Miguel, R., Donert, K. \& Koutsopoulos, K. (Eds.): Geospatial Technologies in Geography Education, Cham: Springer, pp.127-140. 
